\title{
Cleanness evaluation method of remaining dirt on inner surface of narrow tubule by IR spectroscopy
}

\author{
Shouhei Koyama, Yuki Miyauchi, Hiroaki Ishizawa \\ Dept. of Faculty of Textile Science and Technology, Shinshu University, Nagano, Japan
}

Email address:

shouhei@shinshu-u.ac.jp (S. Koyama)

\section{To cite this article:}

Shouhei Koyama, Yuki Miyauchi, Hiroaki Ishizawa. Cleanness Evaluation Method of Remaining Dirt on Inner Surface of Narrow Tubule by IR Spectroscopy. Optics. Vol. 2, No. 1, 2013, pp. 25-31. doi: 10.11648/j.optics.20130201.14

\begin{abstract}
We try to develop a new measurement method for cleanliness evaluation of inner surface of narrow tubule.In this measurement method, we use the infrared spectroscopy and the partial least squares regression (PLSR).The absorption spectrum is measured by using fiber probe and cone mirror.We measure the spectra of the known amount of dirt. We build a calibration curve using these spectra and the PLSR.Next, we measured spectrum of inner surface of narrow tubule, and calculates the amount of remaining this dirt by the calibration curve. This method can be measured in a non-contact and non-destructive. This method can identify the remaining position and the type and amount of dirt.In this paper, we propose this measurement method as new cleanliness evaluation method has overcome the problems that exist in the cleanliness evaluation.
\end{abstract}

Keywords: Cleanness Evaluation, IR Spectroscopy, Optical Fiber Probe And Cone Mirror, Inner Surface Of Narrow Tubule, Remaining Dirt

\section{Introduction}

In the product once used, dirt is remained.To use it again, we must wash the remaining dirt [1].Therefore, a number of authors have investigated about cleaning method and cleaning agent [2] [3].It have been developed many products for cleaning (The washing machine used to a high-pressure jet or ultrasound, the variety of detergents used to acid or alkali, etc).On the other hand, the number of studies of cleanness evaluation method of after cleaning is little [4].Users primarily visually evaluate cleanliness of the products. When the remaining dirt is not visible, user is judged in "no remaining dirt".However, if there is dirt in a position that is not visible is dangerous in this determination.

The product of a complex shape is difficult to wash in perfect.In such a position, it is difficult to evaluate the visually.Furthermore, this location is often remaining dirt.Many products have been used in the field of medicine and food [5].To prevent contamination, cleanliness of each product is demanded in these fields [6].The inner surface of narrow tubule is completely invisible, in particular metallic products [7]. In the present, these products are treating in disposable or only cleaning.As yet, there is no effective cleanliness evaluation method.Therefore, it is demanded to a measuring device which can evaluate the cleanliness of the inner surface of narrow tubule in quickly and easily, their needs are very high.

\section{System design}

\subsection{Measurement System}

There are important points in developing the cleanliness evaluation system of the inner surface of narrow tubule.If it uses a chemical in cleanliness evaluation, we will need to re-wash the chemical.We must not the use of chemicals.It causes scratches and the remaining dirt, if the sensor head hits against the inner surface of narrow tubule. We must be measured by non-contact [8].Moreover, in order to perform cleaning when the remaining dirt is detected, it is necessary to determine the type of dirt [9] [10].

Infrared light is absorbed in a specific wavelength range for each molecule.Therefore, the infrared light is absorbed by the remaining dirt. We can measure the infrared absorption spectrum including information of remaining dirt[11]. The wavelength band of absorption peak of the infrared absorption spectrum differs by type of remaining dirt.We can be qualitative analysis of remaining dirt by wavelength band of absorption peak. 
The amount of absorbed infrared light is proportional to the amount of the sample. The information of the amount of remaining dirt is included in the size of absorbance of absorption peak. Therefore, we can be quantitative evaluation of remaining dirt by this information.

We have developed a cleanliness evaluation system of the inner surface of narrow tubules using the cone mirror and the optical fiber probe by infrared spectroscopy [12]. Measurement system is shown in Fig.1.We use Fourier transform infrared spectrophotometer (FT-IR, SHIMADZU, IRPrestige) in the measurement system.For the infrared light is desired to irradiate directly, we use the optical fiber probe and cone mirror.The optical fiber probe (REMSPEC IMM-07S) composed of 19 fibers (the light source side: 7 and the detector side: 12) made by the chalcogenide glass.As shown in Fig.1, the light source side fiber and the detector side fiber are arranged alternately.Fiber diameter is $500 \mu \mathrm{m}$,optical fiber probe diameter is $5 \mathrm{~mm}$.

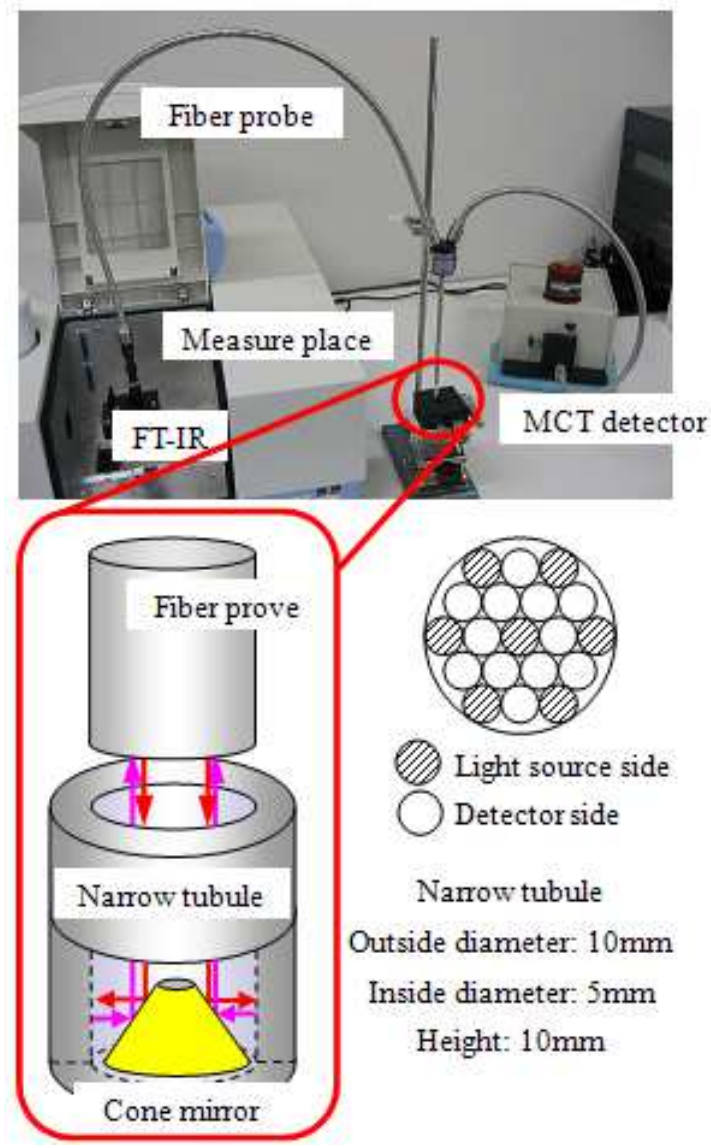

Figure 1. Measurement system for cleanness evaluation.

Broadband infrared light emitted from the light source is interfering by the Michelson interferometer in FT-IR, sent to the optical fiber probe.The interference light from the FT-IR passes the light source side fiber, is irradiated in all direction toward the inner surface of narrow tubule by the cone mirror. Infrared light reflected from the inner surface of the narrow tubule is reflected by the cone mirror again, goes into the detector side fibers. This infrared light is de- tected by the mercury cadmium tellurium detector, appear on the PC as an interferogram signal.This signal transformed by Fourier transformation, we get the spectrum of sample.We measure the pure sample, it is the background signal $\left(\mathrm{I}_{0}\right)$. The absorbance is value of logarithm of the measuring signal $\left(\mathrm{I}_{\mathrm{t}}\right)$ divided by the background (BKG) signal $\left(\mathrm{I}_{0}\right)$. In the absorption spectrum, the horizontal axis is the wavenumber which is the reciprocal of the wavelength, and the vertical axis is absorbance.

\subsection{Samples and Experimental Design}

There are many kinds of material of the narrow tubule.In this paper, it focuses on the metal material which cannot be visual evaluation of inner surface.In the sample preparation, lard and aluminum pipe (Inside diameter: $5 \mathrm{~mm}$, Outside diameter: $10 \mathrm{~mm}$, height: $10 \mathrm{~mm}$ ) are used as the model substance of the human dirt and narrow tubule. The total area of the inner surface of the aluminum pipe is $157 \mathrm{~mm}^{2}$.

Model sample are prepared by the following process.

1. Lard is dissolved in the hexane solution.

2. The aluminum pipe has been kept in the solution for 1 minute.

3. The sample pipe is left in the laboratory for five minutes until the residual hexane is fully evaporated.

4. The dirt on the outer surface of aluminum pipe is wiped off.

The weight of aluminum pipe before and after these processes is measured bythe electronic balance. The weight difference of aluminum pipe is used as the remaining amount of the dirt of reference value per inner surface area $\left(157 \mathrm{~mm}^{2}\right)$.

Cleanliness evaluation of the inner surface of narrow tubule is measured by the following steps.First, cone mirror is placed inside of the clean aluminum pipe, and it is measured the BKG.Next, set up the aluminum pipe with remaining dirt similarly to the BKG measurement. We measure the infrared absorption spectrum of dirt adhering to the entire bottom surface $5 \mathrm{~mm}$ on aluminum pipe. Subsequently, reverse the top and bottom of the aluminum pipe, and measures the rest inner surface. We measure two times in one sample.Two measured absorption spectra are averaged.This average absorption spectrum is used as an infrared absorption spectrum of the sample. In the measurement condition, the resolution is $4 \mathrm{~cm}^{-1}$, the accumulation is 100 times, and the measuring wavenumber range set at 2000 $\mathrm{cm}^{-1} \sim 900 \mathrm{~cm}^{-1}$ (Wave length $5 \sim 11.1 \mu \mathrm{m}$ ).

\subsection{Partial Least Squares Regression (PLSR)}

We are using PLSR for a quantitative analysis.PLSR has not multicollinearity in Multilinear regression (MLR). Measurement accuracy of PLSR is better than Principal Components Regression (PCR) in a small number of factors [13]-[16].In PLSR, the explanatory variables is the absorption spectrum, the objective variable is the reference value of remaining dirt.Assume that errors are present in the objective variable and the explanatory variable.A regression 
equation is calculated after PLS factor is extracted, and new objective variable is calculated.New PLS factor is extracted after a similar calculation by the calculated objective variable and explanatory variable.Add a new PLS factor, and we re-calculate the objective variable.If a number of PLS factors increased, Standard Error of Calibration curve (SEC) is smaller [17].

However, increasing the number of PLS factors too, Standard Error of Prediction (SEP) increases (Over Fitting). We validate the optimal number of PLS factors by Leave-one-out method.The objective variables is calculated by adding a new PLS factor, if the residual is significant before and after in adding PLS factor.If the residual is not significant before and after in adding the PLS factor, select the model that is built before.We use as best calibration model value when the model of this optimal number of factors [18] [19].

\section{Spectrum Measurement}

There are many types of remaining dirt of inner surface of narrow tubule.For example, it is the lipid dirt and protein, etc. We focus on lipid dirt.

In order to measure the infrared absorption spectrum of the lipid stains, we measure the finger lipid dirt.The infrared absorption spectrum of the lipid dirt is shown in Fig.2 (red line). Absorption peaks of $1110 \mathrm{~cm}^{-1}$ and $1458 \mathrm{~cm}^{-1}$ are the vibration by the $\mathrm{CH}$ bonding of the $\mathrm{CH}_{2}$ group. Sharp absorption peak of $1746 \mathrm{~cm}^{-1}$ is the stretching vibration of $\mathrm{CO}$ double bonding, and absorption peak of $1746 \mathrm{~cm}^{-1}$ is the antisymmetric stretching vibration of $\mathrm{CO}$ single bonding. The infrared absorption spectrum of the lard is shown in Fig.2 (blue line). Lard is the excellent model dirt, because absorption spectrum of human lipid dirt is consistent with lard.Therefore, this study uses lard as model lipid dirt.

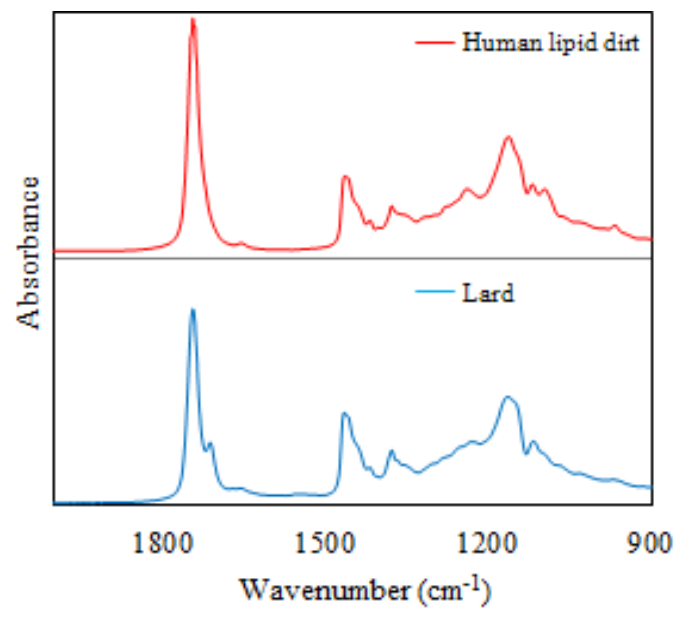

Figure 2.Specrta of lard and human lipid dirt.

\section{Results}

\subsection{Measurement Spectrum of Inner Surface of Narrow Tubule}

Using the development system in this study, we try detection of remaining dirt of inner surface of narrow tubule.Number of samples is five $(0 \mathrm{mg}, 0.08 \mathrm{mg}, 0.20 \mathrm{mg}$, $0.50 \mathrm{mg}$ and $0.71 \mathrm{mg}$ amount of lard), we measure the infrared absorption spectrum of each.These absorption spectra are shown in Fig.3.

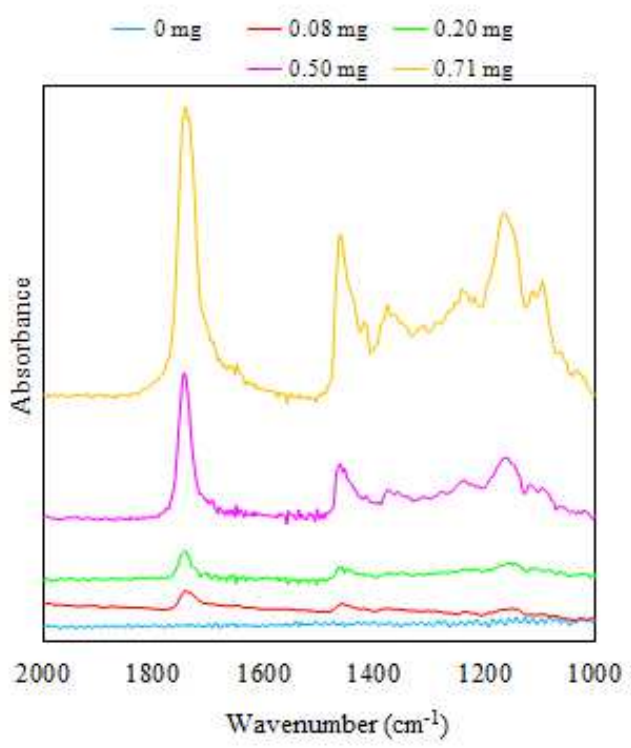

Figure 3. Spectra of $0 \mathrm{mg}, 0.08 \mathrm{mg}, 0.20 \mathrm{mg}, 0.50 \mathrm{mg}$ and $0.71 \mathrm{mg}$ amount of lard.

Absorption peak in the spectrum of $0 \mathrm{mg}$ amount of lard is not seen.In other infrared absorption spectra, absorption peak appeared at $1746 \mathrm{~cm}^{-1}, 1458 \mathrm{~cm}^{-1}, 1167 \mathrm{~cm}^{-1}$ and 1110 $\mathrm{cm}^{-1}$. The wavenumber of these absorption peaks are consistent with the main wavenumber of absorption peak of lard.By this result, it is confirmed to detect remaining dirt of narrow tubule.It can determine the presence or absence of remaining dirt of inner surface of narrow tubule of after cleaning, because shape of the infrared absorption spectrum will vary depending on the presence or absence of dirt.Absorption peaks increase with increasing amount of lard.Therefore, the infrared absorption spectrum has information about the amount of remaining dirt.

In order to accurately extract this information, this study tries to two way analysis. The first is a method to predict the amount of remaining dirt by simple linear regression analysis using absorbance of absorption peak. The second is a method to build a calibration curve by PLSR, substitute the measuring infrared absorption spectrum for calibration curve, and calculate the amount of remaining dirt.We aims to build a good calibration curve for the cleanliness evaluation of the narrow tubule of inner surface by each calculated method.

\subsection{Calibration Curve by Simple Linear Regression.}

We calculate the amount of remaining dirt by build a calibration curve, since the information of the amount of remaining dirt is included in the absorbance of the absorption 
peak.In the experiment, we use a sample of 40 types as shown in Table1.

Table 1. Samples data.

\begin{tabular}{llllll}
\hline No & $\begin{array}{l}\text { Weight } \\
(\mathbf{m g})\end{array}$ & No & $\begin{array}{l}\text { Weight } \\
(\mathbf{m g})\end{array}$ & No & $\begin{array}{l}\text { Weight } \\
(\mathbf{m g})\end{array}$ \\
\hline 1 & 0.13 & 15 & 0.41 & 29 & 0.69 \\
2 & 0.17 & 16 & 0.41 & 30 & 0.71 \\
3 & 0.19 & 17 & 0.43 & 31 & 0.73 \\
4 & 0.20 & 18 & 0.45 & 32 & 0.78 \\
5 & 0.23 & 19 & 0.50 & 33 & 0.80 \\
6 & 0.27 & 20 & 0.54 & 34 & 0.85 \\
7 & 0.28 & 21 & 0.57 & 35 & 0.92 \\
8 & 0.29 & 22 & 0.59 & 36 & 0.94 \\
9 & 0.30 & 23 & 0.63 & 37 & 0.95 \\
10 & 0.32 & 24 & 0.64 & 38 & 0.40 \\
11 & 0.36 & 25 & 0.65 & 39 & 0.63 \\
12 & 0.37 & 26 & 0.66 & 40 & 0.80 \\
13 & 0.38 & 27 & 0.67 & Ave. & 0.52 \\
14 & 0.39 & 28 & 0.68 & & \\
\hline
\end{tabular}

We measure the infrared absorption spectrum of each sample.It analyzed the absorbance at the wavenumber of five absorption peaks $\left(1746 \mathrm{~cm}^{-1}, 1458 \mathrm{~cm}^{-1}\right.$ and $\left.1167 \mathrm{~cm}^{-1}\right)$ of measured spectra, calibration curve is built by simple linear regression analysis. Table 2 shows in the results of the calibration curve for each.By Table 2, there is a significant correlation at all wavenumber of absorption peaks.However, all of coefficient of determination is around 0.5 , these are not a good result.

As this cause, it seems that the effects of noise on spectra and the analyzed in a single absorbance. The $\mathrm{S} / \mathrm{N}$ ratio is very low because the amount of remaining dirt is very small. There is noise on the measured infrared absorption spectrum as Fig.3.By the noise will appear in the absorption peak, it is a significant impact on the results due to the analysis of a single absorbance.We seem a less accurate calibration curve has been constructed, because this effect happened to the measured each spectrum.Therefore, it is expected to be difficult to calculate the amount of the remaining dirt of inner surface of narrow tubule by simple linear regression analysis in a single absorbance.

Table 2. Results by simple linear regression.

\begin{tabular}{llll}
\hline Wavenumber (cm-1) & C.C & C.D & Error (a.u.) \\
\hline 1746 & 0.729 & 0.532 & 0.12 \\
1458 & 0.692 & 0.479 & 0.06 \\
1167 & 0.655 & 0.429 & 0.09 \\
\hline
\end{tabular}

C.C: Correlation coefficient

C.D: Coefficient of determination

\subsection{Calibration Curve by PLSR}

It is difficult to calculate the amount of remaining dirt by a single regression analysis, we calculate the amount of remaining dirt by PLSR.PLSR is suitable for this measurement, because it is constructed the calibration curve on the assumption that the objective variable and the explanatory variables have errors.

The analyzed spectral data are same data in Table 1.To determine the optimal analysis wavenumber range, it analyze the wavenumber band of the three absorption peaks $\left(1800 \mathrm{~cm}^{-1} \sim 1650 \mathrm{~cm}^{-1}, 1500 \mathrm{~cm}^{-1} \sim 1400 \mathrm{~cm}^{-1}, 1200 \mathrm{~cm}^{-1} \sim\right.$ $\left.1000 \mathrm{~cm}^{-1}\right)$, and it analyze by a wavenumber range including the absorption peaks of these $\left(1800 \mathrm{~cm}^{-1} \sim 1000 \mathrm{~cm}^{-1}\right)$.

The results of the calibration curve by PLSR in each wavenumber ranges are shown in Table 3.By these results, there is a significant correlation in the results of most.The model by PLSR is more effective, because there is a correlation equal to or greater when compared to Table 2.In other words, the good calibration curve can be obtained from the analysis of the wide wavenumber range, than analysis in the wavenumber of one absorption peak. The calibration curve in the $1800 \mathrm{~cm}^{-1} \sim 1000 \mathrm{~cm}^{-1}$ range is shown in Fig.4.Correlation coefficient is 0.819 , standard error calibration (SEC) is $0.13 \mathrm{mg}$, the analysis of this wavenumber range is considered to be the most effective.

Table 3. Results of calibration curve by PLSR.

\begin{tabular}{lll}
\hline Range $\left(\mathbf{c m}^{-1}\right)$ & & value \\
\hline \multirow{3}{*}{$1800 \sim 1650$} & Factor & 2 \\
& C.C & 0.724 \\
& SEC $(\mathrm{mg})$ & 0.16 \\
$1500 \sim 1400$ & Factor & 3 \\
& C.C & 0.821 \\
& SEC (mg) & 0.13 \\
$1200 \sim 1000$ & Factor & 2 \\
& C.C & 0.660 \\
& SEC (mg) & 0.17 \\
$1800 \sim 1000$ & Factor & 3 \\
& C.C & 0.819 \\
& SEC (mg) & 0.13 \\
\hline
\end{tabular}

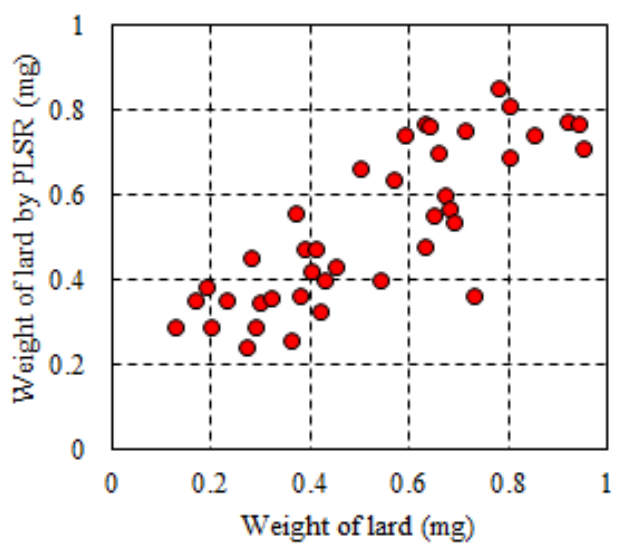

Figure 4. Calibration curve in the $1800 \mathrm{~cm}^{-1} \sim 1000 \mathrm{~cm}^{-1}$ range.

It validates the cleanliness evaluation of inner surface of narrow tubule from this calibration curve.Samples are 
prepared from the 7 kind lard-hexane solutions.Table 4 is shown to the information of sample is prepared by a these solution.It is measured the infrared absorption spectra of these samples.It is substituted these spectra for the calibration curve, calculating the amount of remaining dirt.The prepared sample is weighed the amount of remaining dirt by an electronic balance, evaluate the SEP between the amount of remaining dirt and calculated value from the calibration curve.The results are shown in Fig.5.In the scatter diagram of validation results of the cleanliness evaluation, the horizontal axis is weight of calculated lard by calibration curve, and the vertical axis is weight of measured lard by electric balance.

Table 4. Samples data in validation of calibration curve.

\begin{tabular}{llllll}
\hline No & $\begin{array}{l}\text { Weight } \\
(\mathbf{m g})\end{array}$ & No & $\begin{array}{l}\text { Weight } \\
(\mathbf{m g})\end{array}$ & No & $\begin{array}{l}\text { Weight } \\
(\mathbf{m g})\end{array}$ \\
\hline 1 & 0.00 & 4 & 0.23 & 7 & 0.65 \\
2 & 0.08 & 5 & 0.30 & AVE. & 0.27 \\
3 & 0.18 & 6 & 0.48 & & \\
\hline
\end{tabular}

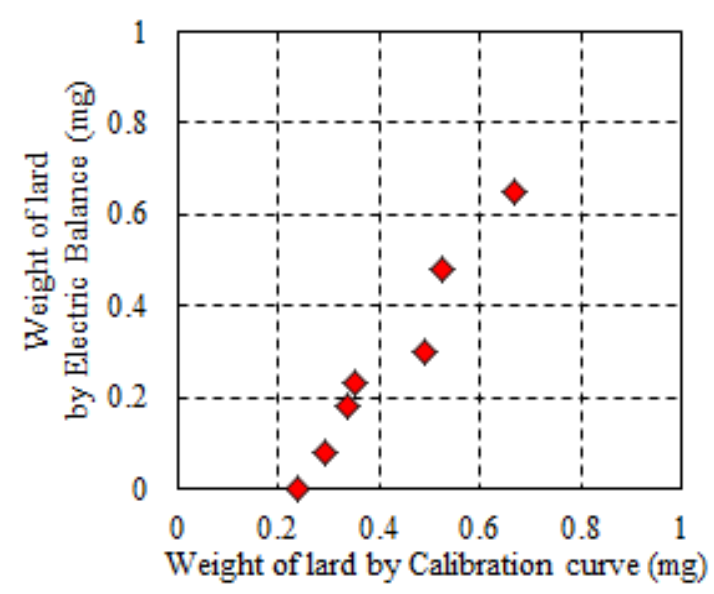

Figure 5. Validation result of calibration curve.

By result of Fig.5, measured value of the remaining dirt and the calculated values by the PLSR is far from $\mathrm{Y}=\mathrm{X}$. The SEP is $0.16 \mathrm{mg}$, which is greater than the minimum amount of remaining dirt.This cause is considered as the information of remaining dirt in the spectrum is buried in the noise, or the baseline of the spectrum varies. Therefore, we must be removed these effects by the spectrum correction, to build a better calibration curve.

\subsection{Calculated the Remaining Dirt by Improved to the Calibration Curve by PLSR}

In order to improve the measurement accuracy of the cleanliness rating, improve the calibration curve. Absorption peak of the small amount of remaining dirt is small.We differentiate the infrared absorption spectrum. The information of small absorption peak becomes clear by the differential.The impact of baseline disappears, because it will see the slope only of the spectrum by differential correction. Therefore, it is expected that much information of re- maining dirt can be extracted.

We differentiate on spectral data of Table1, construct a calibration curve in the $1800 \mathrm{~cm}-1 \sim 1000 \mathrm{~cm}-1$ wavenumber range.It is substituted spectra in Table 4 for the calibrationcurve, calculating the amount of remaining dirt.The results of calibration curve and validation are shown in Fig.6 and Fig.7.The correlation coefficient and SEC of the calibration curve are 0.904 and $0.10 \mathrm{mg}$, these results have been improved. SEP is improved to $0.08 \mathrm{mg}$. Therefore, it is considered that the information of remaining dirt extracts well by differential to the infrared absorption spectrum.From the above results, we confirmed that the measurement method in this study can be measured quantitatively the amount of remaining dirt of inner surface of the narrow tubule.

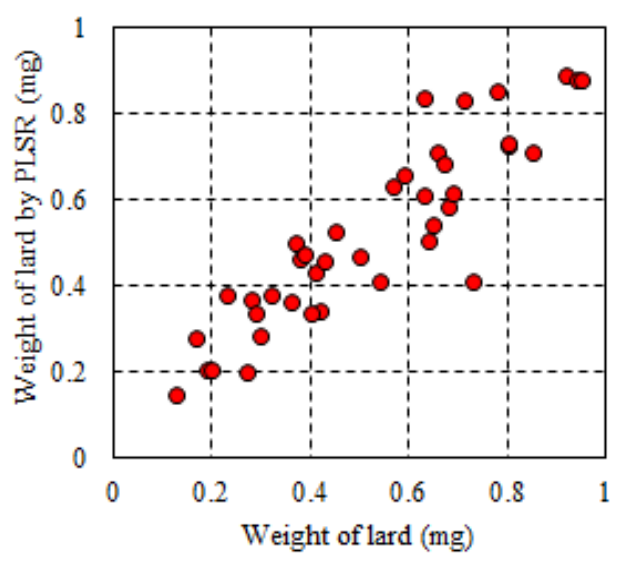

Figure 6. Result of improved calibration curve.

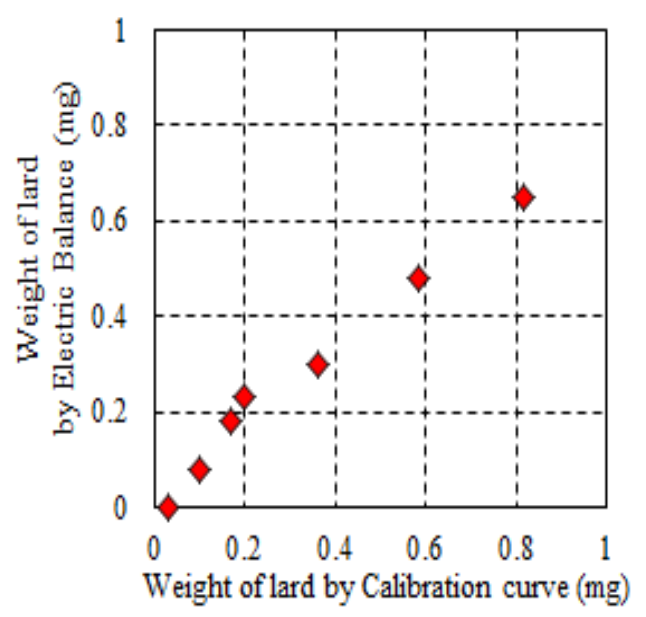

Figure 7. Result of validation by improved calibration curve.

\section{Conclusions}

From the results of experiment, the validity of the cleanliness evaluation measurement system which using to infrared spectroscopy has been shown.The measurement time is about two minutes, it is a very short time. This measurement method is not need re-cleaning, because don't use chemicals.Also, this measurement method can measure the 
non-contact and non-destructive, there are no scratches and dirt.

Future, cone mirror and the fiber probe is united.The sensor head is inserted into the inner surface of narrow tubule, as shown in Fig.8. The sensor head goes forward while monitoring the infrared absorption spectrum of the inside narrow tubule. When the absorption peak of remaining dirt is not detected, we can confirm that the product is cleanly. When the absorption peak of remaining dirt is detected, detection place and qualitative and quantitative evaluation of remaining dirt are measured.If these information are found, and narrow tubule is washed again.If these information are found, and narrow tubule is washed again.Therefore, the cleanliness evaluation of inner surface of narrow tubule which could not be measured can be easil- ly. I believe we will be able to receive a more safety living, since cleanliness evaluation can be.

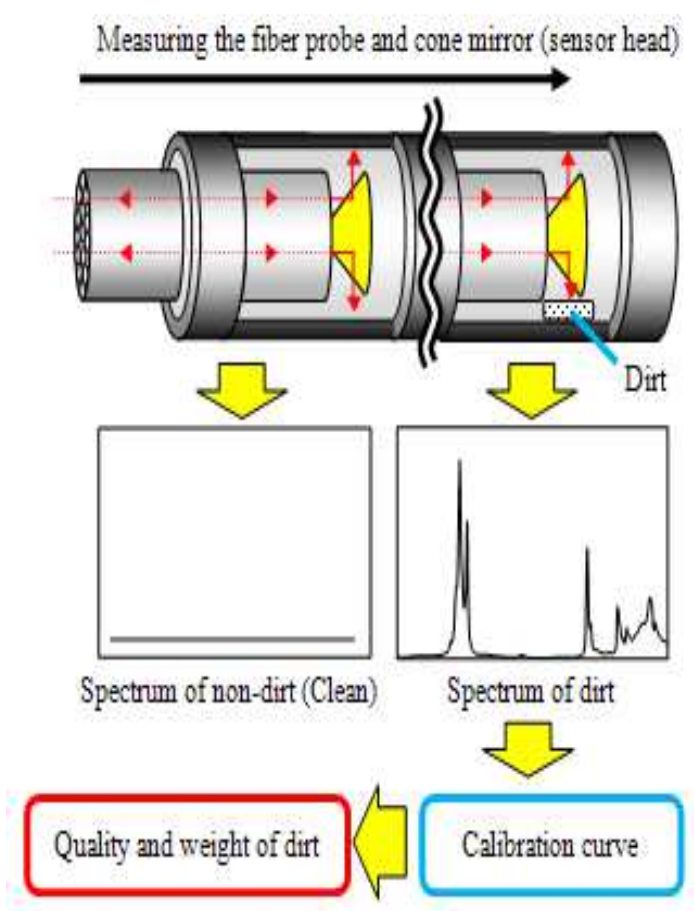

Figure 8. Cleanness evaluation system of inner surface of narrow tubule

From more characteristics, this measurement method has so many advantages, is an excellent cleanliness evaluation.In addition, cleanliness evaluation of inner surface of narrow tubule is also required in the field of medical, food and industrial. This study can be responded to requests sought, we can be proposed as a new cleanliness evaluation method.

\section{References}

[1] Murdoch H., Taylor D., Dickinson J., Walker J. T., Perrett D., Raven N. D. and Sutton J.M., Surface decontamination of surgical instruments, an ongoing dilemma, J. Hosp. Infect, 63(4), 2006, pp.432-438.
[2] R. Ulrika, E. Lars, H. Paul, L. Torbjörn, L. Lisa, L. A. Lisa, M. Eva and R. Klas, A test for cleaning and disinfection processes in a washer-disinfector, Apmis, vol.109, 4, 2001, pp. 299-304.

[3] Jetté L. P., Lambert N.G., Evaluation of two hot water washer disinfectors for medical instruments, Infect Control HospEpidemiol,9(5), 1988, pp.194-199.

[4] Smith G.W., Goldie F., Long S., Lappin D.F., Ramage G. and Smith A.J.,Quantitative analysis of residual protein contamination of podiatry instruments reprocessed through local and central decontamination units, Journal of Foot and Ankle Research, 4(2), 2011.

[5] B. M. Andersen, K.Hochlin and J. P.Daling, Cleaning and Decontamination of Reusable Medical Equipments,Including the use of Hydrogen peroxide Gas Decontamination, J Microbial BiochemTechnol, vol. 4(2), 2012, pp. 057-062.

[6] WHO Guidelineson Hand Hygiene in Health Care, WHO Library Cataloguing-in-Publication Data, World Health Organization 2009.

[7] M. J. Alfa, P. Degagne and N. Olson, Worst-case soiling levels for patient-used flexible endoscopes before and after cleaning, American Journal of Infection Control, vol.27, 5, 1999, pp.392-401.

[8] Baxter R. L., Baxter H. C., Campbell G. A., Grant K., Jones A., Richardson P. and Whittaker G., Quantitative analysis of residual protein contamination on processed surgical instruments, J Hosp Infect, vol.63, 2006, pp.439-444.

[9] M. Shimizu, S. Nakata, R. Fushimi, A. Mizutani, S. Noguchi, M. Monden, Y. Isaka and S. Uraoka, Development of the High Sensitivity Measurement Method for Checking Cleanliness of Medical Instruments, Japanese society of Medical Instrumentation, vol.75, No.1, 2005, pp.28-33.

[10] T. Oda, Y. Hirata and T. Furuta, Evaluation of Cleaning Efficacy and Examination of New Test Soil Model, Japanese society of Medical Instrumentation, vol.76, No.11, 2006, pp.793-797.

[11] D.R. Vij, Handbook of Applied Solid State Spectroscopy, Springer, 2006, pp. 411-450.

[12] S. Koyama, H. Ishizawa, Y. Sakata, T. Gotoh, K. Nakamura, H. Kanai, T. Nishimatsu and E. Toba, Measurement of Remaining Dirt in Medical Instrument Based on IR Spectroscopy and Cone-Shaped Mirror Reflectance, IEEE IMTC, 2005, pp.921-924.

[13] D. M. Haaland and E. V. Thomas, Partial LeastSquares Methods for Spectral Analyses. 1, Relation to Other Quantitative Calibration Methods and the Extraction of Qualitative Information, Analytical Chemistry, vol. 60, No. 11, 1988.

[14] S. Wold, A. Ruhe, H. Wold, and W. J. Dunn, The Collinearity Problem in Linear Regression, The Partial Least Squares (PLS) Approach to Generalized Inverses, SIAM J Sci Stat Comput, 5, 1984, pp. 735-743.

[15] S. Wold, M. Sjostrom and L. Ericksson, PLS-regression, a basic tool of chemometrics, ChemomIntell Lab Syst, 58, 2001, pp. 109-130.

[16] A. Lorber, L. E. Wangen and B. R. Kowalski, A theoretical foundation for the PLS algorithm, Journal of Chemometrics, vol.1(1), 1987, pp. 19-31. 
[17] S. Koyama, Y. Miyauchi and H. Ishizawa, Measurement system of remaining dirt on inner surface of a narrow tubule based on infrared spectroscopy, SICE Annual Conference, 2011, pp.82-86.

[18] Rohman A., Kuwat T., Retno S., Sismindari, Yuny E. and
Tridjoko W., Fourier Transform Infrared Spectroscopy applied for rapid analysis of lard in palm oil, International Food Research Journal,vol.19 (3), 2012, pp.1161-1165.

[19] R. Kramer, Chemometric Techniques for Quantitative Analysis, Marcel Dekker INC, 1998, pp. 131-142. 